ORIGINAL ARTICLE

\title{
Exposure to repeat doses of antenatal glucocorticoids is associated with altered cardiovascular status after birth
}

\author{
L F J Mildenhall, M R Battin, S M B Morton, C Bevan, C A Kuschel, J E Harding
}

Arch Dis Child Fetal Neonatal Ed 2006;91:F56-F60. doi: 10.1136/adc.2004.065300

See end of article for authors' affiliations

\section{Correspondence to:}

Professor Harding, Liggins Institute, Faculty of Medical and Health Science,

University of Auckland,

Private Bag 92019 ,

Auckland, New Zealand;

i.harding@auckland.ac.nz

Accepted 19 August 2005

Published online first

20 September 2005

\begin{abstract}
Objective: To determine if exposure to more than one course of antenatal glucocorticoids is associated with changes in infant blood pressure and myocardial wall thickness in the first month after birth.

Design: Prospective cohort study.

Setting: Tertiary neonatal intensive care unit.

Participants: Mothers who were eligible for but declined to enter a randomised trial of repeated doses of antenatal glucocorticoids (ACTORDS) - that is, who had a singleton, twin, or triplet pregnancy at $<32$ weeks gestation, had received an initial course of glucocorticoids seven or more days previously, and were considered to be at continued risk of preterm birth.

Main outcome measures: Blood pressure daily for the first week then weekly until 4 weeks of age. End diastolic interventricular septal and left ventricular posterior wall (EDIVS and EDLVPW) thickness at 4872 hours after birth.

Results: Thirty seven women were enrolled and delivered 50 infants. Thirty mothers (39 infants) were exposed to one course of glucocorticoids, and seven mothers (11 infants) to more than one course. Blood pressures were higher in the first week after birth in infants exposed to multiple courses of glucocorticoids, and in infants with a latency between last exposure and delivery of less than seven days. Systolic blood pressure on day 1 was $>2$ SD above published normal ranges in $67 \%$ of babies exposed to multiple courses and $24 \%$ of babies exposed to a single course of glucocorticoids $(p=0.04)$. There was no difference between groups in thickness of the EDIVS or EDLVPW. However, 44/50 (88\%) babies had EDIVS and 49/50 (98\%) babies had EDLVPW thickness $>2$ SD above the expected mean for birth weight and gestation. EDIVS but not EDLVPW thickness increased with increasing latency (mean $0.02 \mathrm{~mm} /$ day, $p=0.03$.

Conclusion: Future randomised trials should assess the long term effects of exposure to antenatal glucocorticoids, particularly multiple courses, on the cardiovascular status of the infant.
\end{abstract}

$\mathrm{T}$ he benefits of antenatal glucocorticoids for the preterm neonate include reduction in the risk of respiratory distress syndrome, intraventricular haemorrhage, and neonatal mortality. ${ }^{1}$ However, the greatest benefit is seen in infants born within seven days of treatment. The optimal management of women remaining at risk of preterm birth more than seven days after treatment remains unclear. Administration of repeated courses of antenatal glucocorticoids remains common practice, ${ }^{2}{ }^{3}$ but there is insufficient evidence on the benefits and risks of repeat courses to recommend their use outside the context of randomised trials. $^{4}$

Preterm neonates have also commonly been exposed to glucocorticoids after birth, to prevent or treat chronic lung disease. In this context, the cardiovascular effects of glucocorticoids are well described, and include increased blood pressure and hypertrophic cardiomyopathy. ${ }^{56}$ Both effects are time dependent and resolve after glucocorticoids are discontinued.

There is some evidence that similar effects may be observed after antenatal glucocorticoid exposure. Preterm babies exposed to antenatal glucocorticoids have higher mean blood pressure and less need for inotropic support after birth..$^{7-9}$ However, there are no data on the duration of these effects, or whether there is a greater response with repeated exposure. There are also no human data on possible effects of antenatal glucocorticoids on myocardial hypertrophy. This is particularly important given the evidence from animal studies that prenatal exposure to glucocorticoids can result in raised blood pressure persisting into adulthood. ${ }^{10}$

In view of the uncertainty about the risks and benefits of repeat doses of antenatal corticosteroids, a multicentre randomised controlled trial (ACTORDS) is under way in Australasia. Women who were eligible for, but did not consent to, randomisation to that trial were invited to take part in this study to determine blood pressure and myocardial thickness in babies exposed to at least one course of antenatal glucocorticoids, and to determine the duration of any effects after the last glucocorticoid exposure.

\section{METHODS}

Women with a singleton, twin, or triplet pregnancy at $<32$ weeks who had received an initial course of glucocorticoids beginning seven or more days earlier, and who were at continued risk of preterm birth but had declined participation in ACTORDS were approached for this study. Further courses of glucocorticoids were at the discretion of the woman and her caregiver. Written consent was obtained from the mother before delivery. The Auckland Ethics Committee approved the study.

Blood pressure was measured as soon as possible after birth, daily for the first seven days, and then weekly for four weeks. Three successive measurements were made between

Abbreviations: EDIVS, end diastolic interventricular septal; EDLVPW, end diastolic left ventricular posterior wall 


\begin{tabular}{|c|c|c|c|}
\hline & Single course & Repeat courses & p Value \\
\hline \multicolumn{4}{|l|}{ Mothers } \\
\hline Number & 30 & 7 & \\
\hline Age (years) & $31.6(5.1)(21-45)$ & $32.8(7.7)(21-44)$ & 0.62 \\
\hline Parity & $0.9(1.4)(0-6)$ & $1.6(1.4)(0-4)$ & 0.28 \\
\hline Multiple pregnancy & $7(23.3)$ & $4(57.1)$ & 0.16 \\
\hline Twin & $4(13.3)$ & $3(42.9)$ & \\
\hline Triplet & $3(10.0)$ & $1(14.3)$ & \\
\hline Non-smoker & $26(86.7)$ & $5(71.4)$ & 0.68 \\
\hline Gestation at entry (weeks) & $27.8(1.8)(25-31)$ & $27.3(2.4)(24-30)$ & 0.53 \\
\hline Gestation at last steroid dose (weeks) & $26.5(1.7)(23-30)$ & $29.3(2.6)(25-33)$ & 0.001 \\
\hline Risk for preterm delivery & & & 0.23 \\
\hline Preterm labour & $5(16.7)$ & $1(14.2)$ & \\
\hline Hypertension & $11(36.7)$ & $0(0.0)$ & \\
\hline Rupture of membranes & $8(26.6)$ & $3(42.9)$ & \\
\hline Other & $6(20.0)$ & $3(42.9)$ & \\
\hline \multicolumn{4}{|l|}{ Infants } \\
\hline Number & 39 & 11 & \\
\hline Gestation at birth (weeks) & $31.3(2.2)(27-36)$ & $32.4(3.0)(28-38)$ & 0.15 \\
\hline Birth weight (g) & $1609(446)(755-2870)$ & $1923(581)(1100-2920)$ & 0.07 \\
\hline Female & $20(51.3)$ & $8(72.7)$ & 0.21 \\
\hline Latency (days) & $31.1(15.8)(7-56)$ & $15.5(26.3)(0-90)$ & 0.02 \\
\hline
\end{tabular}

0800 and 1200 hours using a Dinamap XL vital signs monitor (Critikon, Tampa, Florida, USA) and an appropriately sized cuff, with the infant quiet. Mean values were recorded for analysis.

Infants were studied 48-72 hours after birth with M mode echocardiography using a Philips ATL HDI 3000 scanner and $7 \mathrm{MHz}$ probe (Philips Medical Systems, Royal Philips Electronics, Eindhoven, The Netherlands). End diastolic interventricular septal (EDIVS) thickness and end diastolic left ventricular posterior wall (EDLVPW) thickness at the level of the mitral valve leaflets averaged over three cardiac cycles were measured from a parasternal view. ${ }^{11}$ Measurements were undertaken by one investigator (LFJM), who was not aware of the infant's glucocorticoid exposure.

Data were analysed in two ways. Firstly, the characteristics of mothers and infants exposed to single or repeated courses of antenatal glucocorticoids and the cardiovascular outcomes were compared using Student's $t$ tests, factorial analysis of variance, and $\chi^{2}$ tests as appropriate. Secondly, all infants were considered together to determine whether there was any effect of latency on infant cardiovascular outcome, where latency was defined as the interval between the last antenatal glucocorticoid administration and delivery. Latency was dichotomised into $\leqslant 7$ days and $>7$ days, as this is the interval commonly used for repeat courses of antenatal glucocorticoids. Linear mixed models were used to investigate the effects of exposure to single or repeat courses, and the effects of latency, on blood pressure separately for the first week, and the first four weeks. These models adjusted for gestation, sex, and birth weight, and allowed for the clustering of the data from multiple pregnancies and the repeated measures of blood pressure over time. Analyses were conducted using SAS version 9.1 and Statview 5.0 (SAS Institute, Cary, North Carolina, USA). Data are presented as number $(\%)$ or mean (SD) and range.

\section{RESULTS}

Thirty seven mothers were enrolled and delivered 50 infants. Thirty (81\%) mothers had been exposed to a single course of glucocorticoids, and seven to more than one course (four ( $11 \%)$ to two courses and three $(8 \%)$ to three). The initial glucocorticoid course comprised two doses of $11.4 \mathrm{mg}$

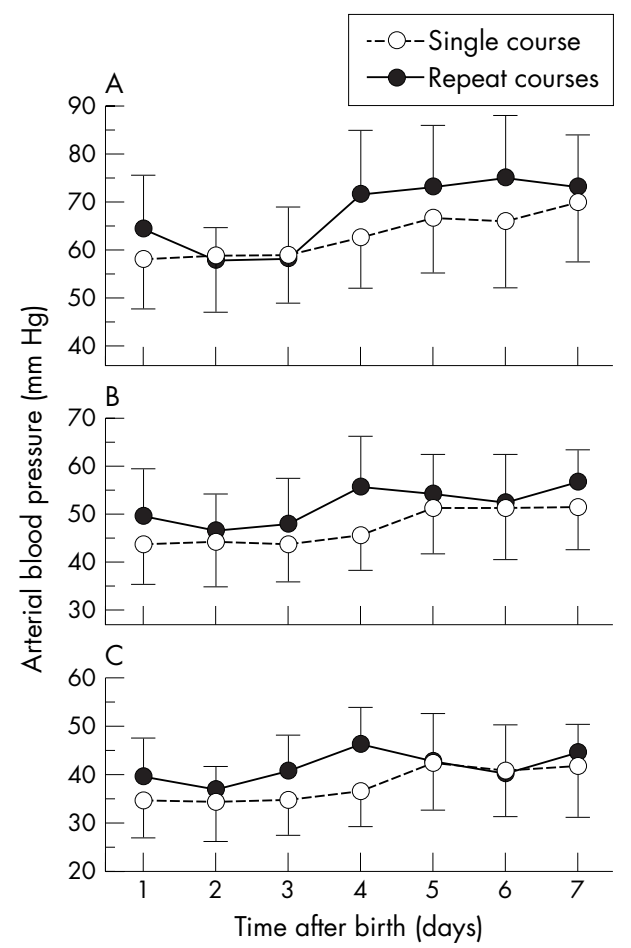

Figure 1 Systolic (A), mean (B), and diastolic (C) blood pressures in the first week after birth in infants exposed to single and repeat courses of antenatal glucocorticoids. Data are mean (SD) for 39 infants exposed to single and 11 exposed to repeat courses. Comparison between groups over whole week: systolic $p=0.08 ;$ mean $p=0.05$; diastolic $p=0.05$.

betamethasone 24 hours apart in 31 women and two doses $12 \mathrm{mg}$ dexamethasone 24 hours apart in six women. There were no significant differences in maternal or neonatal characteristics, or the aetiologies of threatened preterm labour, between the groups exposed to single or repeat courses (table 1).

Eight infants were born $\leqslant 7$ days after exposure to the last dose of glucocorticoids (short latency group) and $42>7$ days 
Table 2 Blood pressure $(\mathrm{mm} \mathrm{Hg})$ over the first four weeks after birth in infants exposed to single and repeat courses of glucocorticoids

\begin{tabular}{|c|c|c|c|c|}
\hline & \multicolumn{2}{|l|}{ Single course } & \multicolumn{2}{|c|}{ Repeat courses } \\
\hline & Mean (SD) & No & Mean (SD) & No \\
\hline \multicolumn{5}{|l|}{ Day 1} \\
\hline Systolic & $52.5(9.2)$ & 25 & $65.7(11.2)$ & 9 \\
\hline Diastolic & 32.9 (8.3) & 25 & $35.9(7.6)$ & 9 \\
\hline Mean & $40.6(8.5)$ & 25 & $47.7(9.4)$ & 9 \\
\hline \multicolumn{5}{|l|}{ Week 1} \\
\hline Systolic & $68.4(12.8)$ & 29 & 74.9 (13.8) & 11 \\
\hline Diastolic & $42.4(12.0)$ & 29 & $45.3(6.8)$ & 11 \\
\hline Mean & 51.9 (12.2) & 29 & $56.8(9.4)$ & 11 \\
\hline \multicolumn{5}{|l|}{ Week 2} \\
\hline Systolic & 74.0 (13.7) & 26 & 79.7 (13.2) & 11 \\
\hline Diastolic & $46.8(10.9)$ & 26 & $48.1(11.3)$ & 11 \\
\hline Mean & $56.3(11.5)$ & 26 & $59.1(11.2)$ & 11 \\
\hline \multicolumn{5}{|l|}{ Week 3} \\
\hline Systolic & $71.9(11.2)$ & 28 & 79.0 (11.7) & 9 \\
\hline Diastolic & $40.6(9.1)$ & 28 & $50.3(10.5)$ & 9 \\
\hline Mean & $52.8(11.4)$ & 28 & $60.1(12.4)$ & 9 \\
\hline \multicolumn{5}{|l|}{ Week 4} \\
\hline Systolic & $82.0(11.4)$ & 26 & $84.3(8.1)$ & 11 \\
\hline Diastolic & 44.9 (9.0) & 26 & 46.7 (9.5) & 11 \\
\hline Mean & $58.4(9.6)$ & 27 & $60.1(10.4)$ & 11 \\
\hline
\end{tabular}

There are no significant differences between the two groups over weeks 1-4.

after exposure (long latency group). Seven of the eight infants in the short latency group were exposed to repeat courses of glucocorticoids. Mean latency was two weeks longer in the single course group, although the range was 756 days compared with 0-90 days in the repeat course group (table 1).

\section{Blood pressure}

Blood pressure measurements throughout the first four weeks were positively associated with gestational age $(\mathrm{p}=$ 0.03 ) and birth weight $(p=0.05)$. Blood pressures were higher in the first week, but not subsequently, in infants exposed to repeat courses of glucocorticoids than those exposed to a single course (table 2, fig 1). When compared with published normal ranges, ${ }^{12}$ systolic blood pressure on day 1 was $>2$ SD above the expected mean in 6/9 (67\%) infants in the repeat course group and 6/25 (24\%) infants in the single course group $\left(\chi^{2}, \mathrm{p}=0.04\right)$.

When the group was considered as a whole, blood pressures were higher in the first week after birth in infants who experienced a latency of $\leqslant 7$ days compared with those with longer latency (fig 2). There appeared to be no further effect of latency on blood pressure after this time.

\section{Myocardial thickness}

EDIVS thickness tended to increase with gestational age at delivery $(p=0.07)$. EDLVPW thickness was not significantly related to birth weight or gestational age.

Mean EDIVS and EDLVPW thickness did not differ between infants exposed to single or repeat courses of glucocorticoids (EDIVS 5.0 (1.3) v 5.3 (1.4) mm; EDLVPW 5.5 (1.4) v $5.4(1.2) \mathrm{mm}$ ). However, 44/50 infants had EDIVS thickness and 49/50 had EDLVPW thickness $>2$ SD above the mean expected for birth weight and gestation. ${ }^{13-15}$ The proportion of abnormal measurements did not differ between the two glucocorticoid exposure groups.

EDIVS thickness was positively associated with the latency duration, with an average increase of $0.02 \mathrm{~mm} /$ day of latency $(p=0.04)$. This association was no longer significant after adjustment for birth weight and gestational age $(0.019 \mathrm{~mm} /$ day, $\mathrm{p}=0.16)$. There was no association between EDLVPW thickness and latency $(\mathrm{p}=0.56)$.

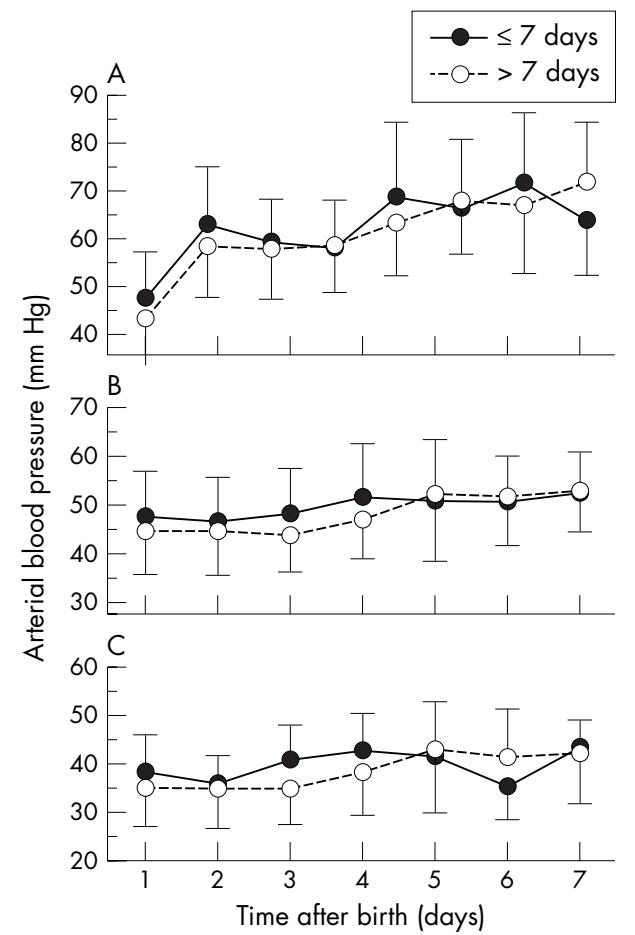

Figure 2 Systolic (A), mean (B), and diastolic (C) blood pressures in the first week after birth in infants experiencing short ( $\leqslant 7$ days) or long (>7 days) latency since last dose of antenatal glucocorticoids. Data are mean (SD) for eight infants who experienced short latency and 42 who experienced long latency. Comparison between groups: systolic $p=$ 0.07 ; mean $p=0.03$; diastolic $p=0.03$.

\section{DISCUSSION}

We report for the first time an association between exposure to repeat courses of antenatal glucocorticoids and blood pressure and myocardial thickness in the neonate. Exposure to repeat courses of glucocorticoids was associated with higher infant blood pressure in the first week after birth. Although there was no difference in myocardial thickness between infants exposed to single and repeat courses of 
glucocorticoids, most infants we studied had EDIVS and EDLVPW thickness greater than previously published norms. The long term significance of these cardiovascular effects of exposure to antenatal glucocorticoids is uncertain but of considerable interest.

Previous studies have reported that antenatal glucocorticoid exposure increases mean blood pressure and decreases the need for inotrope or volume support in the first 2448 hours after birth in very preterm infants. ${ }^{78}$ In sheep, even low dose hydrocortisone administration to the mother results in a substantial rise in fetal blood pressure. ${ }^{16}$ Furthermore, glucocorticoids given to preterm neonates to prevent or treat chronic lung disease result in a prompt and sustained rise in blood pressure, which resolves gradually once glucocorticoids are withdrawn. ${ }^{17}$ In this study we found that blood pressure was raised for the first week after delivery in infants exposed to repeat courses of glucocorticoids before birth. Whether higher blood pressure was associated with exposure to increasing numbers of courses of glucocorticoids could not be determined, as too few infants were exposed to more than two courses.

We found that blood pressure was also higher in the first week in infants who had experienced a latency of less than seven days between their last glucocorticoid exposure and delivery. This suggests that the most obvious effects of antenatal glucocorticoids on infant blood pressure may be relatively short lived. Blood pressure is raised for at least 14 days after discontinuation of postnatal glucocorticoids, ${ }^{17}$ but the much higher doses of glucocorticoids for much longer periods may contribute to this prolonged effect. Unfortunately it was not possible in our study to distinguish the effects of latency from the effects of dose, as entry criteria for this study included a latency of at least seven days since initial exposure to glucocorticoids. Thus inevitably almost all of the infants who eventually experienced a short latency between glucocorticoid exposure and delivery were those exposed to repeat courses of glucocorticoids. This difficulty in distinguishing between the effects of latency and of dose, and hence in resolving the question of the duration of any effect on blood pressure, will remain a problem in future randomised studies for the same reason.

We found that a third of infants in this study had high systolic blood pressure according to published norms and that this was more common in infants exposed to repeat courses of glucocorticoids. The definition of "normal" blood pressure in preterm babies is problematic, and few data exist for the smallest babies. We used a recent publication from the east coast of North America in the early 1990s. This did not document the use of antenatal glucocorticoids, but it seems likely that it was low at that time.

To our knowledge the effect of antenatal glucocorticoid exposure on myocardial thickness in humans has not been previously reported. The hypertrophic effects of postnatal glucocorticoid exposure on cardiac wall thickness are well described.5 ${ }^{6151819}$ Cardiac wall thickness has also been reported to increase by $40 \%$ in fetal sheep exposed to only 10 days of low dose maternal cortisol infusion. ${ }^{16}$ We found no evidence of a dose-response effect on myocardial thickness, in contrast with evidence of a dose-response at the much higher doses used in preterm infants after birth. ${ }^{15}$ Our findings may relate to the much lower dose used, or to fundamental differences in the effects of glucocorticoids on the developing heart before and after birth.

Contrary to our expectations, we found that the effects of glucocorticoid exposure on myocardial thickness increased with increasing latency since last exposure. However, persistent myocardial hypertrophy has been reported in preterm neonates several weeks after discontinuation of dexamethasone. ${ }^{19}$ Other studies of postnatal steroids have shown resolution two to three weeks after discontinuation of treatment. ${ }^{20}$ No infants in our study had any signs of left ventricular outflow obstruction.

Both myocardial thicknesses measured were greater in almost all infants than published norms. ${ }^{13-15}$ We found no single publication reporting normal cardiac measurements for EDLVPW and EDIVS thicknesses across the range of gestations and weights in our sample. Of the publications we used, one listed no antenatal exclusion criteria, and the other two excluded infants of diabetic mothers. Only one article described antenatal steroid use (dexamethasone), ${ }^{15}$ and reported no effect on septal or posterior wall thickness.

Our finding that a large proportion of infants exposed to one or more courses of antenatal glucocorticoids have increased blood pressure and myocardial thickness in the neonatal period raises questions about the possible long term cardiovascular consequences of antenatal glucocorticoid exposure. There are now substantial animal data showing effects of fetal glucocorticoid exposure on blood pressure and cardiac development. In fetal sheep, exposure to a single course of dexamethasone early in gestation increased blood pressure into adulthood. ${ }^{21}$ Fetal rats exposed to increased maternal glucocorticoids throughout pregnancy had increased blood pressure after birth. ${ }^{22}$ Antenatal glucocorticoid exposure has also been implicated in cardiac myocyte hypertrophy and early adulthood degeneration. ${ }^{23}$ Long term follow up of infants enrolled in randomised trials of antenatal glucocorticoids will be required to determine whether any cardiovascular effects persist beyond infancy, and whether they have any later health consequences.

This study cohort was well defined, in that all subjects met criteria for entry into the ACTORDS study, and all data were collected prospectively and analysed by investigators who did not know the details of antenatal glucocorticoid exposure. The cohort is small, and not all measurements were able to be made on all babies. However, this would tend to reduce rather than increase the power of the statistical analysis, and thus is unlikely to explain our findings.

This study has shown that exposure to antenatal glucocorticoids is associated with changes in blood pressure and myocardial thickness after birth. These results require confirmation by larger randomised trials, and strongly suggest that future studies should examine cardiovascular outcomes, not only in the neonatal period but later in childhood and through to adulthood.

\section{What is already known on this topic}

- Glucocorticoids can cause increased blood pressure and myocardial hypertrophy in preterm babies

- Babies exposed to a single course of antenatal glucocorticoids have increased blood pressure at birth

\section{What this study adds}

- Blood pressures were higher in the first week after birth in infants exposed to multiple courses of antenatal glucocorticoids, and in infants born less than seven days after last exposure

- Most exposed babies had increased myocardial wall thickness 


\section{ACKNOWLEDGEMENTS}

This work was funded in part by the Health Research Council of New Zealand and the New Zealand Child Health Research Foundation. We thank all the families whose infants took part in the study, and Elizabeth Robinson for statistical advice. We are grateful for the help and support of Professor Caroline Crowther and the ACTORDS study team, University of Adelaide, Adelaide, Australia.

\section{Authors' affiliations}

L F J Mildenhall, Kidz First Middlemore Hospital, Auckland, New Zealand

S M B Morton, Liggins Institute, University of Auckland

M R Battin, C Bevan, C A Kuschel, J E Harding, Newborn Services, National Women's Health, Auckland District Health Board, Auckland, New Zealand

Competing interests: none declared

\section{REFERENCES}

1 Crowley P. Prophylactic corticosteroids for preterm birth. Cochrane Pregnancy and Childbirth Group. Cochrane Database Syst Rev, 2004;2.

2 Brocklehurst P, Gates S, McKenzie-McHarg K, et al. Chamberlain G. Are we prescribing multiple courses of antenatal corticosteroids? A survey of practice in the UK. Br J Obstet Gynaecol 1999;106:977-9.

3 Mclaughlin KJ, Crowther CA. Repeat prenatal corticosteroids: who still recommends their use and why? Aust NZ J Obstet Gynaecol 2003;43:199-202.

4 McLaughlin KJ, Crowther CA, Walker N. Harding JE. Effects of a single course of corticosteroids given more than 7 days before birth: a systematic review, Aust NZ J Obstet Gynaecol 2003;43:101-6.

5 Werner JC, Sicard RE, Hansen TW, et al. Hypertrophic cardiomyopathy associated with dexamethasone therapy for bronchopulmonary dysplasia. J Pediatr 1992;120:286-91.

6 Brand PL, van Lingen RA, Brus F, et al. Hypertrophic obstructive cardiomyopathy as a side effect of dexamethasone treatment for bronchopulmonary dysplasia. Acta Paediatr 1993;82:614-17.
7 Moise AA, Wearden ME, Kozinetz CA, et al. Antenatal steroids are associated with less need for blood pressure support in extremely premature infants. Pediatrics 1995;95:845-50.

8 Demarini S, Dollberg S, Hoath SB, et al. Effects of antenatal corticosteroids on blood pressure in very low birth weight infants during the first 24 hours of life. J Perinatol 1999; 19:419-25.

9 Elimian A, Figueroa R, Spitzer AR, et al. Antenatal corticosteroids: are incomplete courses beneficial? Obstet Gynecol 2003;102:352-5.

10 Seckl JR. Glucocorticoid programming of the fetus; adult phenotypes and molecular mechanisms. Mol Cell Endocrinol 2001;185:61-71.

11 Sahn DJ, DeMaria A, Kisslo J, et al. Recommendations regarding quantitation in M-mode echocardiography: results of a survey of echocardiographic measurements. Circulation 1978;58:1072-83.

12 Zubrow AB, Hulman S, Kushner $\mathrm{H}$, et al. Determinants of blood pressure in infants admitted to neonatal intensive care units: a prospective multicenter study. Philadelphia Neonatal Blood Pressure Study Group. J Perinatol 1995; 15:470-9.

13 Solinger R, Elbl F, Minhas K. Echocardiography in the normal neonate. Circulation 1973;47:108-18.

14 Walther FJ, Siassi B, King J, et al. Echocardiographic measurements in normal preterm and term neonates. Acta Paediatr Scand 1986;75:563-8.

15 Skelton R, Gill AB, Parsons JM. Reference ranges for cardiac dimensions and blood flow velocity in preterm infants. Heart 1998;80:281-5.

16 Jensen EC, Gallaher BW, Breier BH, et al. The effect of a chronic maternal cortisol infusion on the late-gestation fetal sheep. J Endocrinol 2002; 174:27-36

17 Marinelli KA, Burke GS, Herson VC. Effects of dexamethasone on blood pressure in premature infants with bronchopulmonary dysplasia. J Pediatr 1997; 130:594-602

18 Evans N. Cardiovascular effects of dexamethasone in the preterm infant. Arch Dis Child Fetal Neonatal Ed 1994;70:F25-30.

19 Bloomfield FH, Knight DB, Harding JE. Side effects of 2 differen dexamethasone courses for preterm infants at risk of chronic lung disease: a randomized trial. J Pediatr 1998;133:395-400.

20 Zecca E, Papacci P, Maggio L, et al. Cardiac adverse effects of early dexamethasone treatment in preterm infants: a randomized clinical trial. J Clin Pharmacol 2001:41:1075-81.

21 Dodic M, Moritz K, Wintour EM. Prenatal exposure to glucocorticoids and adult disease. Arch Physiol Biochem 2003;111:61-9.

22 Benediktsson R, Lindsay RS, Noble J, et al. Glucocorticoid exposure in utero: new model for adult hypertension. Lancet 1993;341:339-41.

23 de Vries WB, van der Leii FR, Bakker JM et al. Alterations in adult rat heart after neonatal dexamethasone therapy. Pediatr Res 2002;52:900-6. 